\title{
Bilateral herpes simplex keratitis in a patient with chronic graft-versus-host disease
}

\author{
Takahiko Hayashi' \\ Misaki Ishioka ${ }^{2}$ \\ Norihiko Ito' \\ Yoko Kato' \\ Hisashi Nakagawa ${ }^{3}$ \\ Hiroshi Hatano ${ }^{4}$ \\ Nobuhisa Mizuki' \\ 'Department of Ophthalmology, \\ Yokohama City University School \\ of Medicine, Yokohama, Kanagawa, \\ Japan; ${ }^{2}$ Ryogoku Eye Clinic, Tokyo, \\ Japan; ${ }^{3}$ Tokushima Eye Clinic, \\ Higashimurayama-shi, Tokyo, Japan; \\ ${ }^{4}$ Lumine Hatano Eye Clinic, Fujisawa, \\ Fujisawa-shi, Kanagawa, Japan
}

\begin{abstract}
Purpose: To describe a case of bilateral herpes simplex keratitis accompanying chronic graft-versus-host disease (GVHD).

Design: Observational case report.

Case report: An 11-year-old boy with myelocytic leukemia underwent allogeneic bone marrow transplantation. He developed symptoms of the skin, eyes, and mouth, and lip biopsy indicated chronic GVHD. Persistent keratitis with corneal filaments and neovascularization was noted in both eyes. Sodium hyaluronate, autoserum, and $0.1 \%$ fluorometholone eyedrops were instilled for approximately 2 years to treat this keratitis, and there were no other ocular changes. Bilateral herpes simplex keratitis developed with geographic ulcers after topical betamethasone therapy, but responded to acyclovir ointment.
\end{abstract}

Conclusions: Herpes keratitis should be considered in the differential diagnosis of bilateral keratitis in patients with reduced immunocompetence. During the course of chronic GVHD, corneal herpes may occur, so ocular treatment with topical corticosteroids should be managed by an ophthalmologist to monitor sight-threatening conditions such as corneal herpes.

Keywords: chronic graft-versus-host disease, bone marrow transplant, corneal herpes, bilateral herpes simplex keratitis, dry eyes

Typically, herpes simplex keratitis is unilateral, and the incidence of bilateral keratitis is reported to range from $1 \%$ to $10 \%$ (Ostler 1976).

Here, we present a patient with bilateral herpetic keratitis that developed against the background of chronic graft-versus-host disease (GVHD). Robinson and colleagues (1991) previously reported bilateral herpes simplex keratitis in a patient with GVHD, but they did not clearly state whether the condition was acute or chronic.

\section{Case report}

An 11-year-old boy was diagnosed with myelocytic leukemia in February 2002. There was no relevant family history or past history. After pretreatment with total body irradiation (12 Gy), allogeneic bone marrow transplantation was performed on September 20, 2002 (Day 0). He developed symptoms of the skin, eyes, and mouth, and lip biopsy indicated chronic GVHD in December 2002 (minor salivary glands showed lymphocyte infiltration and interstitial fibrosis). His cutaneous symptoms were dryness and erythema of the skin. From 2003 to the present, keratitis with corneal filaments and neovascularization has been noted persistently in both eyes (Figure 1). From 2003 to September 2005, sodium hyaluronate, autoserum, and $0.1 \%$ fluorometholone eye drops were used for the treatment of this bilateral keratitis. His best-corrected visual acuity was 20/25 OD and 25/25 OS. Although ocular and cutaneous lesions suggested extensive GVHD, his liver function was normal and there were no gastrointestinal or 


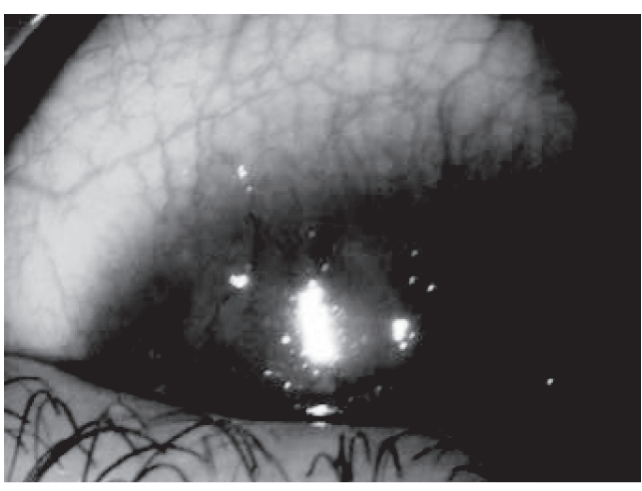

Figure I Photographs of the anterior segment.

Figure I.I From 2003 to the present, both eyes have shown bilateral keratitis with filaments and neovascularization.

respiratory symptoms. The ocular lesions were not severe keratitis sicca, which is typical of chronic GVHD, but the presence of corneal vascular invasion and filamentary keratitis strongly suggested abnormal of tear fluid. Apart from keratitis, there were no other ocular changes. His immunosuppressant therapy was tapered smoothly and oral administration of FK 506 was stopped in August 2004.

Worsening of keratitis in the left eye was noted on September 12, 2005, and topical betamethasone sodium phosphate was instilled twice daily. On September 15th, a geographic ulcer appeared in the left eye (Figure 1.2). Corneal herpes was diagnosed and the ulcer was healed by October 25th using acyclovir ointment.

On February 7th, a geographic ulcer was detected in the right eye (Figure 1.3). Corneal herpes was diagnosed again and was treated with acyclovir ointment, resolving within a week. Since then, recurrence has not been observed. Both eyes had corneal epithelial lesions and there was no parenchymal involvement, iritis, or endothelitis.

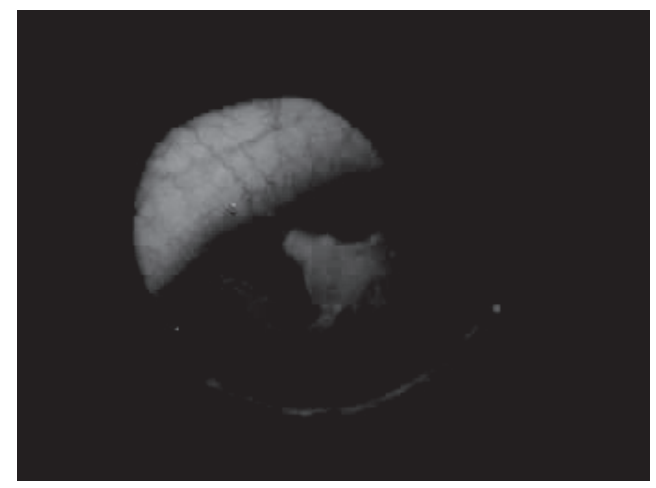

Figure I.2 Geographic ulcer in the left eye, which appeared on September I5th.

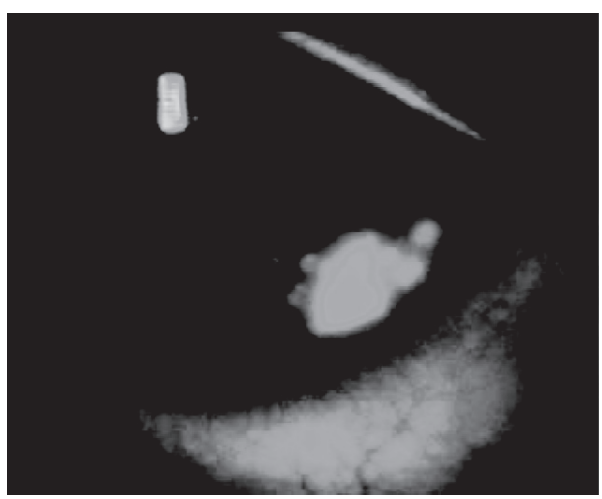

Figure I.3 Geographic ulcer in the right eye, which appeared on February 7th.

Tear fluid from the patient was positive for herpes simplex virus type 1 (HSV-1) on polymerase chain reaction (PCR) analysis (Figure 2).

We performed PCR at the second onset with a cut-off value of 10 plaque-forming units (PFU)/ml. HSV-1, HSV-2, and varicella-zoster virus (VZV) were examined by PCR of tear fluid specimens using the following specific primers (HSV-1: GTTAGGGAGTTGTTCAATCATAAGCT and TCGGCCATCTTGAGAGAGGCATCC, HSV-2: GTCGGTGTGGTGTTCGGTCATAAGCT and GGCTGAATGTGGTAAACACGCTTC, VZV: GGGCGAAATGTAGGATATAAAGGAATCGCGGCTTGTTGTTTGTCTAAT).

The right eye had a typical geographic ulcer, which was clinically diagnosed with herpes and treated. It healed before examinations could be done for diagnosis. At the onset of the second ulcer, we tried to perform immunostaining and PCR using a brush specimen from the lesion. However, the patient was a child and we could not obtain such a specimen. Assessment of corneal sensation, which is essential to diagnose corneal herpes, was also not permitted. The bestcorrected visual acuity after healing of the geographic ulcers was $20 / 25$ OD and $25 / 25$ OS.

\section{Conclusions}

Although rare, bilateral herpetic keratitis has been diagnosed in young patients with atopy (Easty et al 1975), and it has been reported in patients with GVHD (Robinson and Newton 1991). The complication rate is higher in patients with bilateral herpetic keratitis than in patients with unilateral disease (Wilhilmus et al 1981).

GVHD is a major complication of allogeneic bone marrow transplantation that occurs due to the reactivity of transplanted immunocompetent cells against host cells. The 


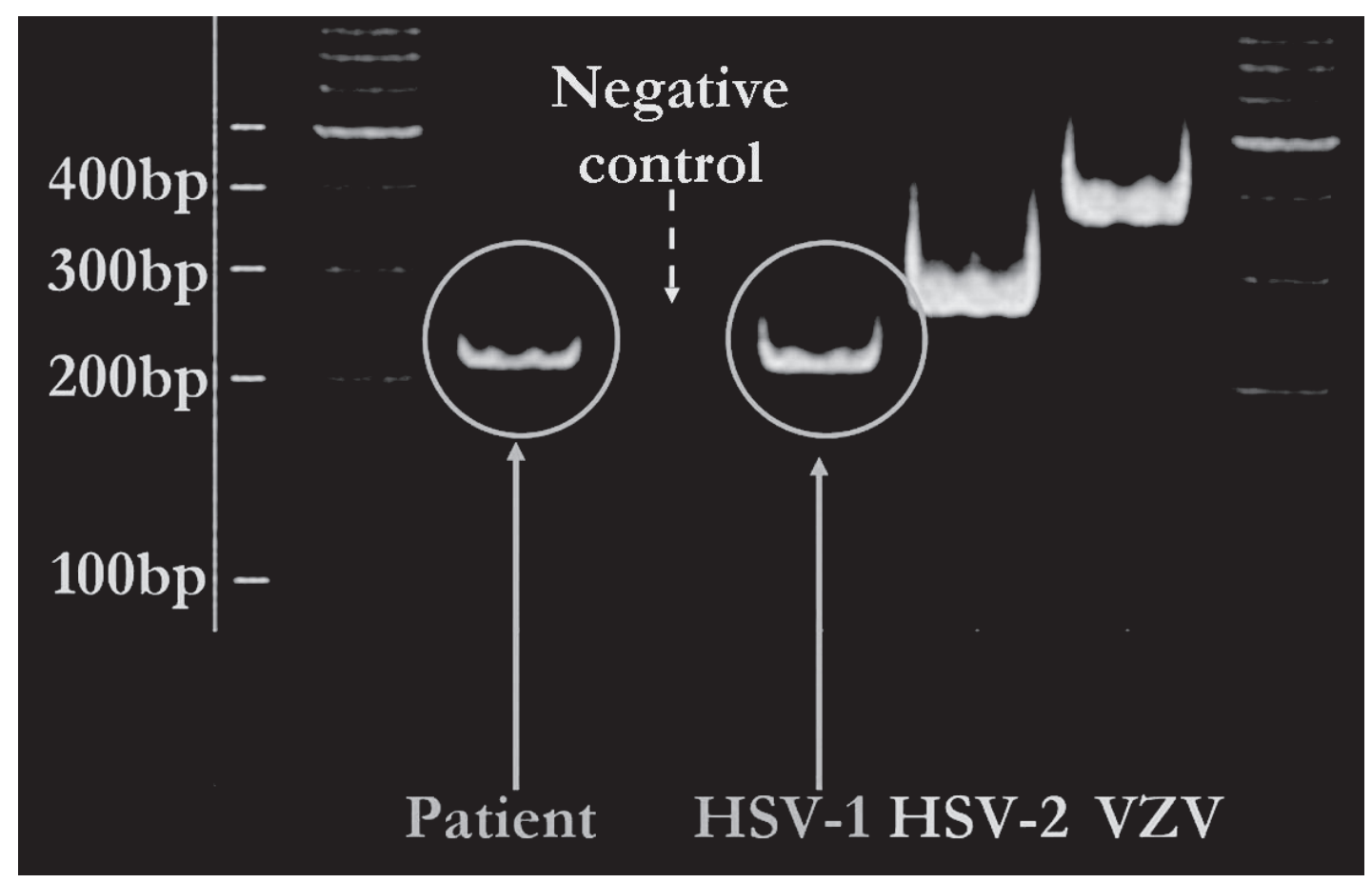

Figure 2 Polymerase chain reaction. Herpes simplex virus type I was positive in the tear fluid. The negative control was shown as a blank PCR test.

incidence of GVHD is $50 \%$ to $70 \%$, and it is classified as acute or chronic. The most frequent ocular manifestation of chronic GVHD is dry eye. Effective treatments for this syndrome include preservative-free artificial tears, corneal protection agents, autoserum eye drops, punctal plugs, and therapeutic contact lenses. In addition, the use of topical ocular corticosteroids is effective for the treatment of conjunctivitis, considering that a conjunctival pseudomembrane can be observed in patients with acute GVHD (Robinson et al 2004) or filamentary keratitis.

The present patient did not have severe dry eyes with lack of tears, which is typical of chronic GVHD, but had atypical changes with filamentary keratitis and vascular invasion of the cornea. Thus, this case suggests that keratoconjunctivitis associated with GVHD can show diversity.

In conclusion, our case suggests that herpetic keratitis should be considered in the differential diagnosis of bilateral keratitis in patients with altered immunity, and shows that the occurrence of corneal herpes is a possibility in chronic GVHD. Therefore, treatment of ocular symptoms with topical corticosteroids in patients with GVHD should be managed by an ophthalmologist to monitor sight-threatening conditions such as corneal herpes.

\section{References}

Easty D, Entwistle C, Witcher J, et al. 1975. Herpes simplex keratitis and keratoconus in the atopic patient. A clinical and immunological study. Trans Ophthalmol Soc UK, 95:267.

Ostler HB. 1976. Herpes simplex: the primary infection. Surv Opthalmol, 21:91-9.

Robinson MR, Lee SS, Rubin BI, et al. 2004. Topical corticosteroid therapy for cicatricial conjunctivitis associated with chronic graft-versus-host disease. Bone Marrow Transplant, 33:1031-5.

Robinson MJ, Newton C. 1991. Bilateral herpes simplex keratitis in a patient with graft-vs-host disease. Am J Ophthalmol, 112:468-9.

Wilhilmus KR, Falcon MG, Jones BR. 1981. Bilateral herpetic keratitis. Br J Opthalmol, 65:385. 
